\title{
L'abbaye de Cluny III : étude de la façade gothique
}

Juliette Rollier-Hanselmann, Camille Collomb et Gaëlle Ferdek

\section{(2) OpenEdition}

\section{Journals}

Édition électronique

URL : https://journals.openedition.org/cem/11974

DOI : $10.4000 /$ cem. 11974

ISSN : 1954-3093

\section{Éditeur}

Centre d'études médiévales Saint-Germain d'Auxerre

\section{Édition imprimée}

Pagination : 203-221

ISSN : 1623-5770

\section{Référence électronique}

Juliette Rollier-Hanselmann, Camille Collomb et Gaëlle Ferdek, «L'abbaye de Cluny III : étude de la façade gothique », Bulletin du centre d'études médiévales d'Auxerre | BUCEMA [En ligne], 15 | 2011, mis en ligne le 28 septembre 2011, consulté le 22 septembre 2022. URL : http://journals.openedition.org/ cem/11974; DOI : https://doi.org/10.4000/cem.11974

\section{Ce document a été généré automatiquement le 22 septembre 2022.}

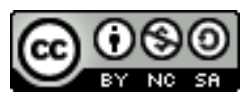

Creative Commons - Attribution - Pas d'Utilisation Commerciale - Partage dans les Mêmes Conditions 4.0 International - CC BY-NC-SA 4.0

https://creativecommons.org/licenses/by-nc-sa/4.0/ 


\title{
L'abbaye de Cluny III : étude de la façade gothique
}

\author{
Juliette Rollier-Hanselmann, Camille Collomb et Gaëlle Ferdek
}

1 Si l'histoire du déclin de Cluny a été étudiée par les historiens ${ }^{1}$, l'évolution de la sculpture gothique n'a pas encore suscité de véritables recherches ${ }^{2}$. La documentation témoigne d'une façade monumentale, ornée de plusieurs sculptures figuratives et d'une grande rose, dont il ne reste malheureusement que quelques dizaines de fragments. Une première analyse archéologique des élévations a été réalisée, ainsi qu'une étude des fragments issus de la façade, de manière à orienter les travaux de restitution en 3D de cette partie de l'abbaye ${ }^{3}$. Une étude des stratigraphies picturales a révélé deux couches picturales, dont la plus ancienne comporte des dorures.

\section{Construction de la façade gothique}

2 Il convient de replacer la construction de la façade de Cluny III dans son contexte architectural. Comme l'ont démontré les archéologues qui l'ont étudiée au cours du $\mathrm{xx}^{\mathrm{e}}$ siècle, l'abbatiale est élevée d'est en ouest ${ }^{4}$. L'avant-nef vient se greffer à la façade de la nef et suit le même principe de construction vers l'Occident. La façade est probablement achevée sous l'abbatiat de Roland de Hainaut (1220-1228)

La documentation concernant l'élévation des tours Barabans est pauvre, comme nous le verrons par la suite. Selon Kenneth John Conant, historien et archéologue américain de la première moitié $\mathrm{du} \mathrm{xx}^{\mathrm{e}}$ siècle, leur construction n'est pas simultanée. L'archéologie permettra de confirmer ou d'infirmer cette hypothèse.

4 Toujours selon le chercheur américain, l'abbé Eudes de la Perrière (1432-1456/1457) est responsable de l'installation de marches d'approche et d'un hémicycle agrémenté d'une croix monumentale en pierre. C'est peut-être à cette époque que le portail de l'église reçoit l'appentis entre les deux Barabans.

5 La construction du palais abbatial par l'abbé Jean de Bourbon (1465-1485) nécessita la mise en place d'un couloir d'accès à la tour Baraban nord. On lui attribue aussi 
certaines restaurations, peut-être dans les petits collatéraux - bassas voltas eiusdem ecclesiae $e^{6}$. L'abbé Jacques d'Amboise (1485-1510) fit construire son propre pavillon (actuelle mairie) et le dessin d'Étienne Martellange (1617) montre déjà un jardin à la française, peut-être dû à cet abbé qui avait des attaches en Touraine.

La vente de la majeure partie de l'abbaye le 24 avril 1798 marque le début des démolitions. Tous les vitraux sont brisés le 16 juillet, puis ceux de la grande rose de la façade. Démunie de ses barres de fer, la rose s'effondre quelques jours plus tard. Le 19 juillet l'appentis recouvrant le portail est détruit? ${ }^{7}$.

7 La destruction de l'avant-nef a lieu en août 1798, et les tours Barabans perdent leurs toitures cette même année. Le démantèlement des deux grandes tours a lieu en juin 1809 pour la tour nord et en octobre 1810 pour la tour sud.

\section{Interprétation des documents}

8 Plusieurs documents graphiques montrent l'abbaye dans son ensemble depuis la face nord, ce qui apporte des indications concernant l'état de la façade gothique ${ }^{8}$. Le dessin de Jean-Baptiste Lallemand ${ }^{9}$, daté vers 1773 , est le document le plus précis et le plus complet que nous ayons concernant l'aspect de la façade depuis l'ouest (fig. 1).

Fig. 1 - J. B. Lallemand, dessin de la façade gothique (musée d'art et d'archéologie-Ochier, Cluny).

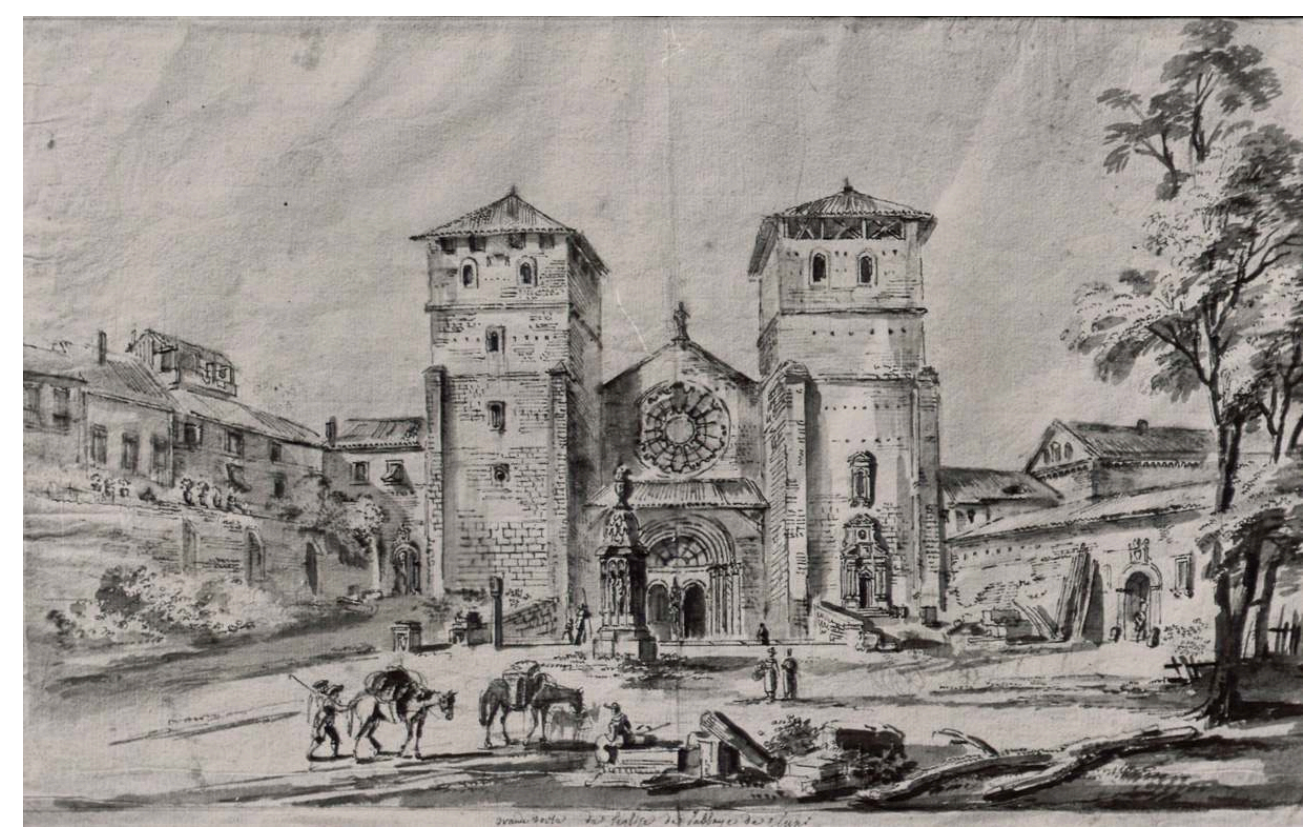

Ce dessin très intéressant indique qu'une demi-rose à six pétales surmontait la double porte d'entrée et qu'une grande rose occupait le mur pignon. Le portail était soutenu par un trumeau central, mais le dessin est trop petit $(16 \times 9 \mathrm{~cm})$ pour donner davantage de détails en ce qui concerne les sculptures. La tour sud comporte également des remaniements tardifs, notamment la porte monumentale due à Richelieu (1629-1642). 
Fig. 2 - F. van Riesamburgh, esquisse du revers de façade (Treille/Conant).

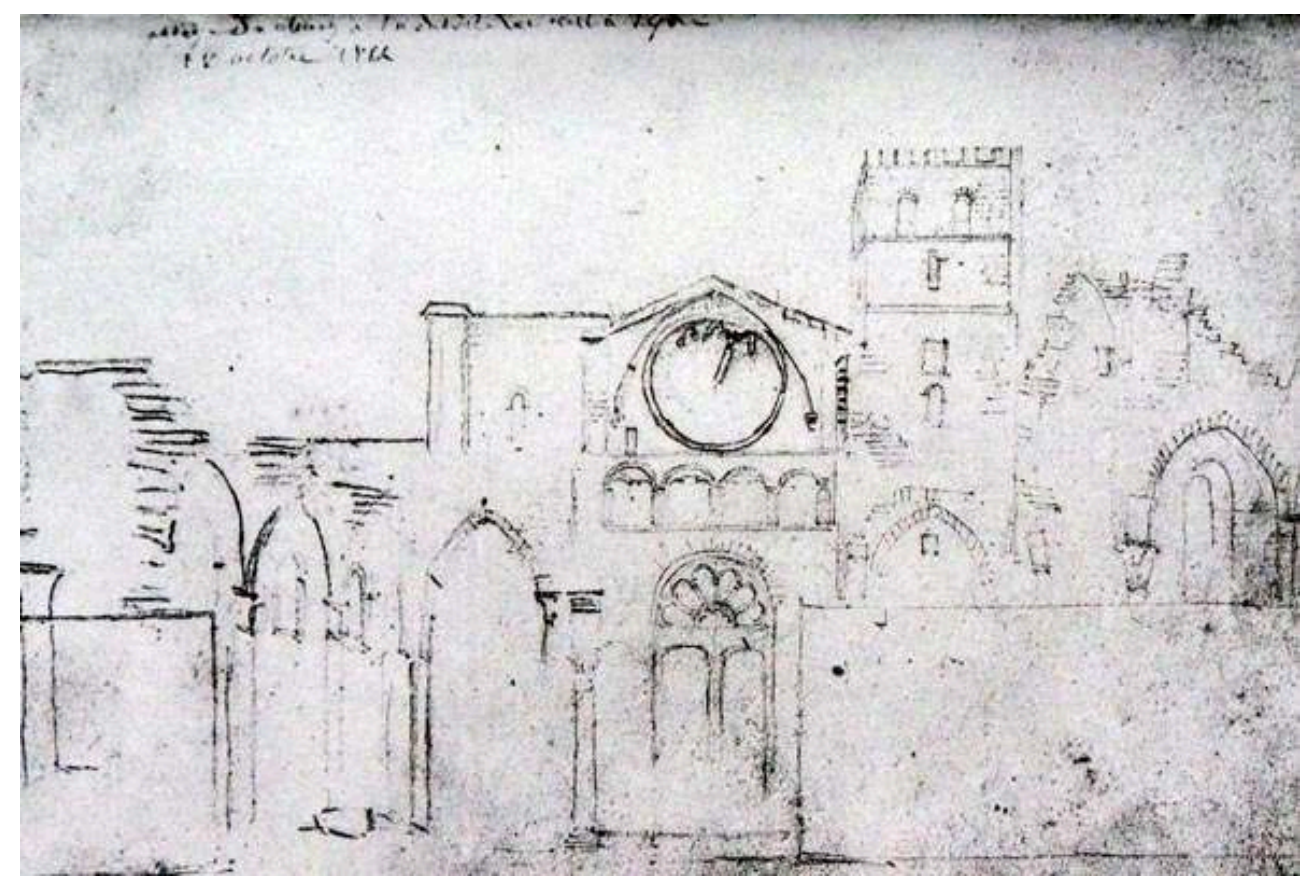

L'esquisse de Fabien Van Riesamburgh ${ }^{10}$, datée de 1814, montre le revers de la façade, en partie effondré (fig. 2). Le dessinateur se situe à l'intérieur de l'église et reproduit l'intégralité de la demi-rose, qui comporte alors six lobes. Au-dessus du portail se trouvent quatre arcatures trilobées et la grande rose s'est déjà effondrée. Seuls quelques remplages subsistent misérablement. Un dessin d'Émile Sagot $-1^{\text {re }}$ moitié du $\mathrm{XIX}^{\mathrm{e}}$ siècle ${ }^{11}$ - présente la façade de l'abbaye, à travers la porte d'honneur, où nous distinguons une rose monumentale.

11 Le portail gothique est connu par deux témoignages oculaires du XVII ${ }^{\mathrm{e}}$ siècle. Un manuscrit intitulé «Fondation de la Sacrée Abbaye de Cluny $»^{12}$, conservé à la Société Éduenne à Autun, datant de 1710, le décrit en ces termes : « (...) cette abbaye (...) ayant deux fort belles tours quarrées au milieu desquelles est située son entrée qui est un portail assez simple où il y a trois grandes figures de pierre, scavoir saint Jean, st Pierre, et saint Paul (...) ».

Le témoignage le plus précis est celui de Benoît Dumolin $(1713-1798)^{13}$, qui donne de nombreux détails et des mesures précises :

«Le portail du vestibule [= avant-nef] est dans le goût gothique; il a environ 26 pieds d'hauteur sur la largeur de 16, ce qui en marque évidemment l'irrégularité. Les jambages sont ornés de chaque côté de quatre colonnes en pierre, isolées, dont la première à droite et à gauche est coupée horizontalement en manière de niches. Dans la droite est placée une figure en pierre, haute d'environ six pieds, sous l'image de saint Jean Évangéliste; Saint Étienne sous une figure parallèle à la première, remplit la niche sur la gauche. Le milieu de la porte est partagé par une colonne perpendiculaire, de niveau aux collatérales, aussi coupée en manière de niche, ornée de la statue de saint Pierre, semblable en tours et hauteurs aux premières figures.

La porte du vestibule est à deux battants en bois, taillée en sculpture, et ornée de trente figures en relief. Les cintres du portail sont surbaissés; des cintres au contour s'élève une demi-rose en vitraux, à cinq colonnes en pierre, placée sur un massif haut, d'environ trois pieds, appuyé sur les cintres. La façade du massif est 
peinte et ornée de trois figures de pierre en relief, celle du centre est l'image de la Vierge, les deux figures collatérales représentent des anges.

Le grand cintre du portail [= tympan] est orné de moulures, qui naissent du niveau des colonnes qui en ornent les flancs. De l'une ou l'autre tour qui terminent la largeur du portail il y a trente pieds... le portail... couvert par un appentis, autrefois décoré par un lambris peint, formé suivant le contour du portail. Au-dessus de l'appentis qui couronne le portail, on voit une rose qui remplit le vide de l'une à l'autre tour ; cette rose est dans son plein cintre, elle forme un niveau d'environ trente pieds de diamètre, composé de 20 branches qui naissent de la circonférence d'une rose plus petite qui forme le centre. La première, les branches de deux en deux terminent à leurs extrémités un centre taillé en trèfle ; le contour du grand centre est formé par une moulure massive en manière de quart-devant, placé entre deux tours creuses. La rose est couronnée par un massif en chevron brisé, élevé au niveau du couvert du vestibule, à l'extrémité du chevron brisé on voit la figure d'un bénédictin en aube, l'encensoir à la main ; cette figure est en pierre haute d'environ 4 pieds et demi, placée sur un piédestal gravé uni. »

La description de Philibert Bouché de la Bertillère (entre 1789 et 1816) n'est qu'une reprise du texte de Benoît Dumolin. En fait, la destruction de l'abbaye est déjà bien avancée en 1811, ce qui oblige l'auteur à recourir à une description plus ancienne.

Quelques cartes postales anciennes permettent encore de connaître l'évolution et les dégradations de la façade, avec la mise en place d'une rue traversant l'avant-nef.

Plusieurs dizaines de dessins, de planches et de calculs remarquables ont été réalisées par l'archéologue américain K. J. Conant pour tenter de reconstituer la façade disparue (fig. 3). Un fonds de plus de 10000 documents a été numérisé, de manière à le rendre accessible pour les chercheurs. Un tri de ce fonds est actuellement en cours.

Fig. 3 - K. J. Conant, restitution de la façade gothique (musée d'art et d'archéologie-Ochier, Cluny).

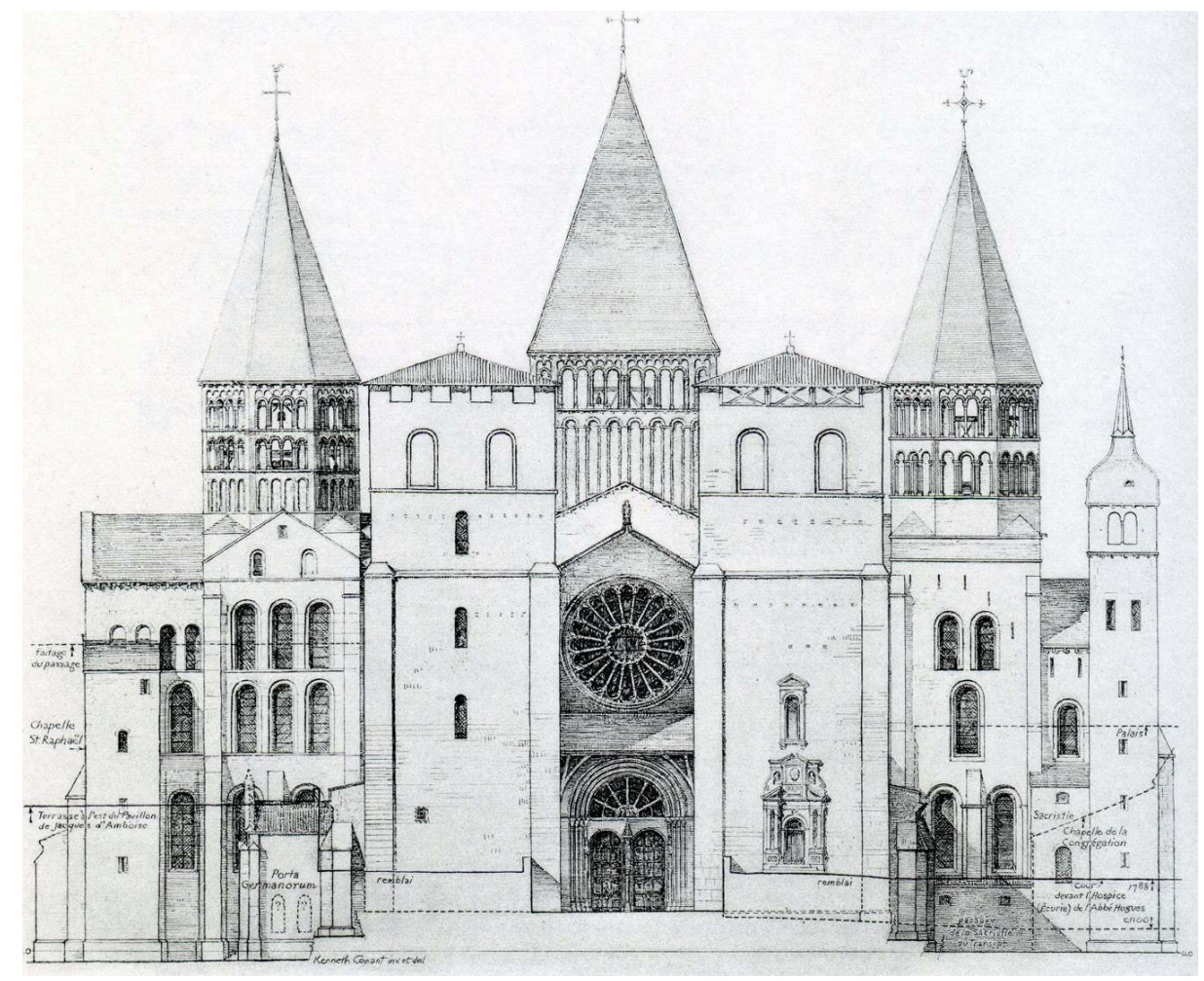




\section{Étude archéologique de la façade gothique}

L'épaisseur du massif de façade est de 2,15 m, auquel il faut ajouter la profondeur des piédroits $(1,80 \mathrm{~m})$. Le soubassement et les bases des piédroits sont encore en place, ainsi que le départ de l'arc surplombant la porte (fig. 4 et 5 ).

Fig. 4 - Cluny III, façade gothique, vestiges du piédroit gauche (cl. J. Rollier).

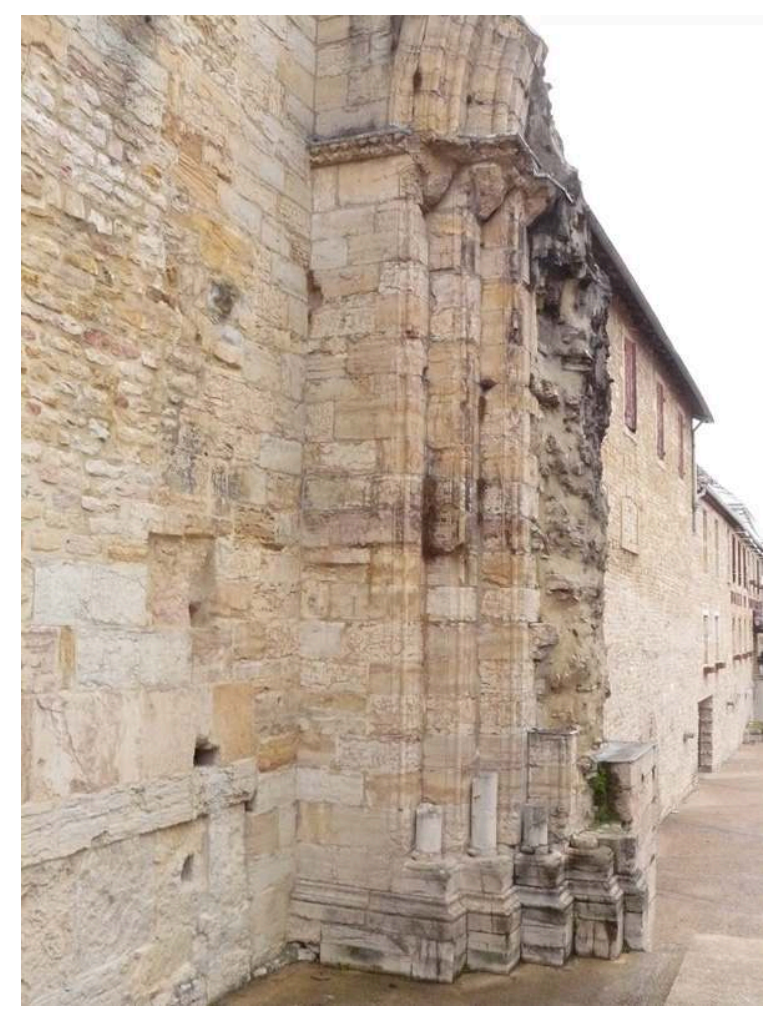


Fig. 5 - Cluny III, façade gothique, vestiges du piédroit gauche (cl. J. Rollier).

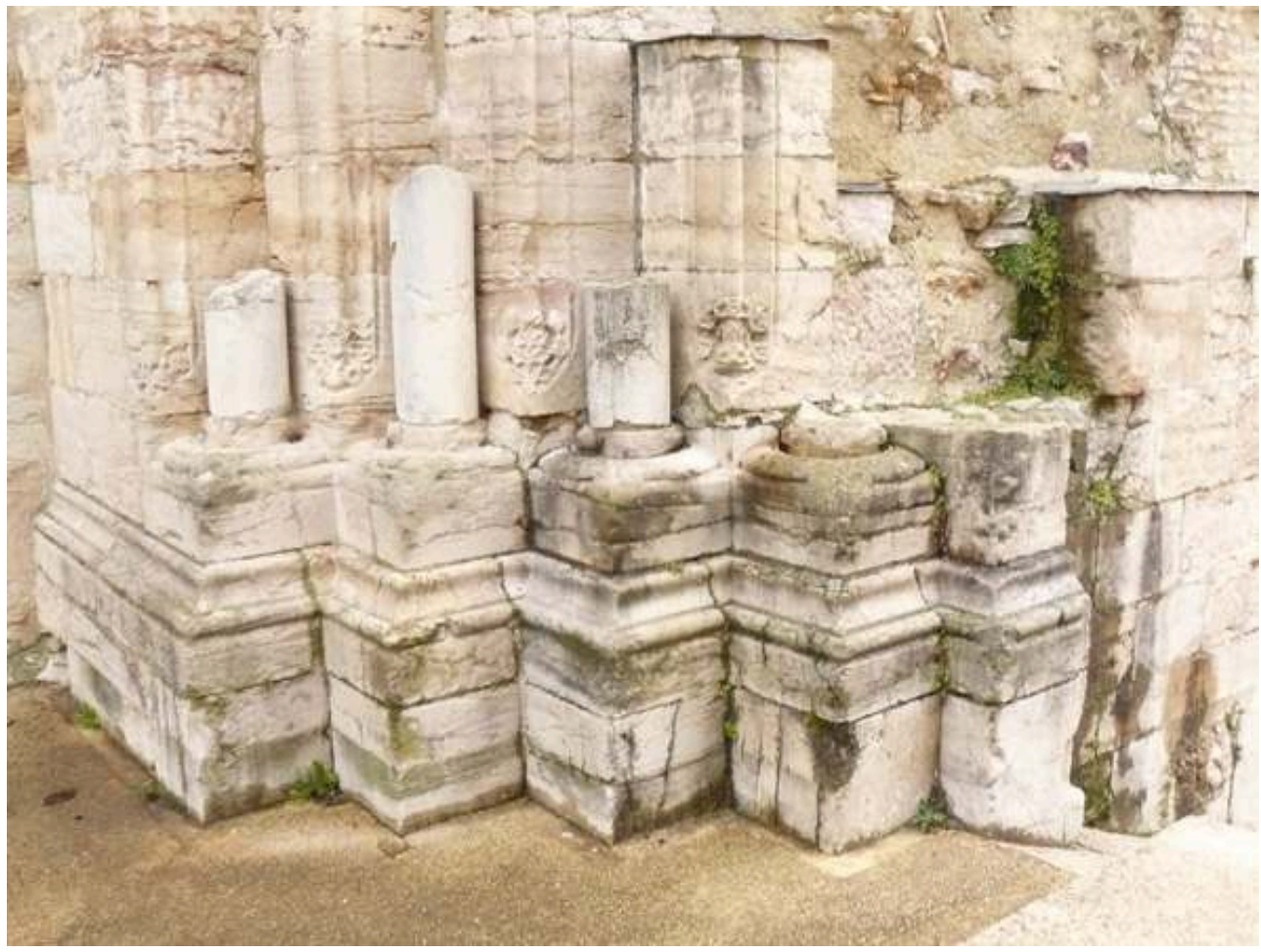

17 Le calcaire à entroques, de teinte beige-rosé, est une roche très résistante qui est utilisée dans l'avant-nef uniquement ${ }^{14}$. Des tracés préparatoires et des marques d'outils sont visibles à plusieurs endroits (fig. 10). Les parements conservent également des restes de badigeon, blanc et jaune, très altérés par les intempéries. 
Fig. 10 - Cluny III, façade gothique, piédroit droit, détail (cl. J. Rollier).

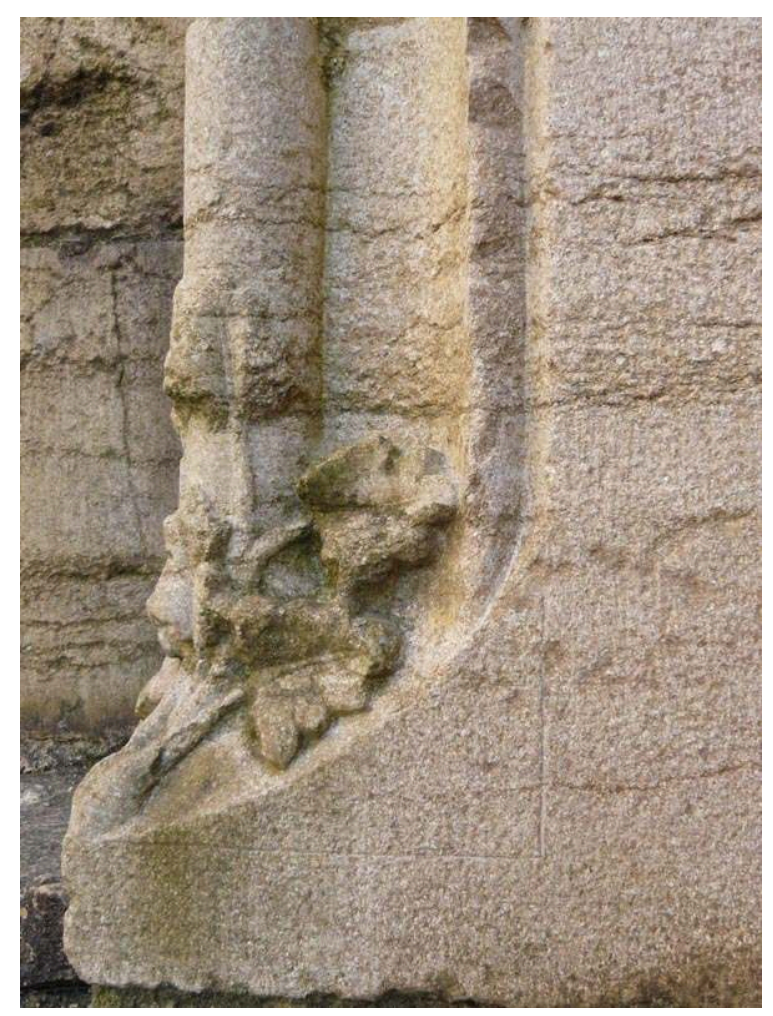

18 Les différentes campagnes de fouilles archéologiques menées successivement par K. J. Conant, de 1928 à 1950, et Gilles Rollier ${ }^{15}$, entre 1988 et 1990, ont mis au jour de nombreux fragments lapidaires. Le remblai de l'avant-nef représente $2000 \mathrm{~m}^{3}$ de terre dont $1500 \mathrm{~m}^{3}$ ont été triés au printemps 1988, d'où sont issus plus de 6000 fragments sculptés. Le tri de ce matériel (400 caissettes) a conduit à l'établissement d'un inventaire de 170 fragments gothiques, pour la plupart de petites et moyennes dimensions (5 à $80 \mathrm{~cm}$ de long). Les autres fragments sont issus des autres parties de l'avant-nef : façade romane, piliers, chapiteaux, frise, arcatures, baies, etc.

19 Les éléments sculptés qui subsistent encore sur les vestiges du portail gothique apportent également des repères : motifs végétaux des bases, colonnettes, frise située en haut du piédroit (fig. 4-5). Ces éléments ont permis de trier plus facilement les fragments et de les regrouper par type. Les feuilles grasses et les feuilles de vigne appartiennent au répertoire de notre façade, éléments que nous retrouvons aussi sur la demi-rose (fig. 8). 
Fig. 8 - Cluny III, élément de la demi-rose (cl. J. Rollier).

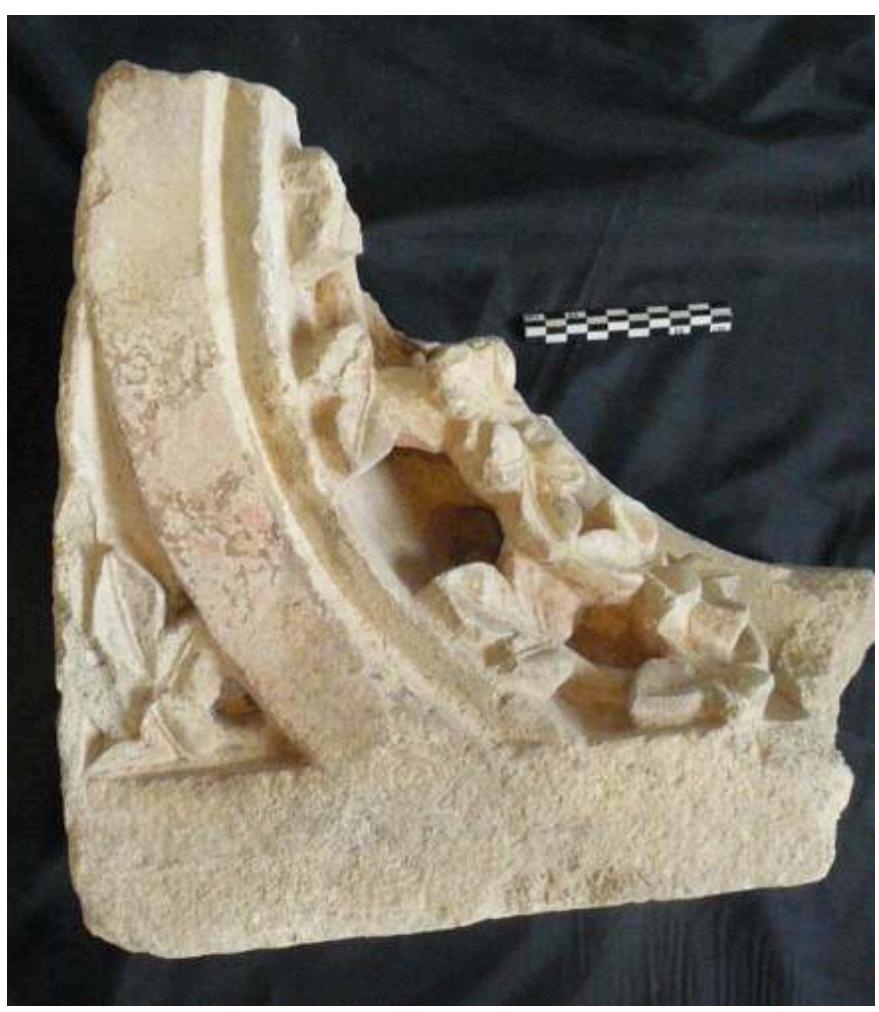

Un coussinet et un groupe de deux chapiteaux ${ }^{16}$, autrefois situés au sommet du piédroit de droite, sont également conservés. Les dimensions et l'aspect des piédroits sont ainsi relativement bien connus. Malheureusement nous n'avons pas localisé d'éléments appartenant aux parties figuratives des piédroits - saint Pierre, saint Étienne et saint Jean Évangéliste - mentionnés dans le témoignage de Benoît Dumolin. D’ailleurs, cet auteur pense reconnaître saint Étienne alors que le document de la Société Éduenne parle de saint Paul, ce qui paraît plus logique à Cluny. Ces éléments ont malheureusement disparu, mais signalons tout de même qu'il subsiste une belle tête portant une mitre - haut. $26,5 \times$ larg. $17 \times$ prof. $16,5 \mathrm{~cm}^{17}$-, de provenance incertaine, sculptée dans un calcaire gris plus fin. 
Fig. 9 - Cluny III, dais central du portail gothique (cl. J. Rollier).

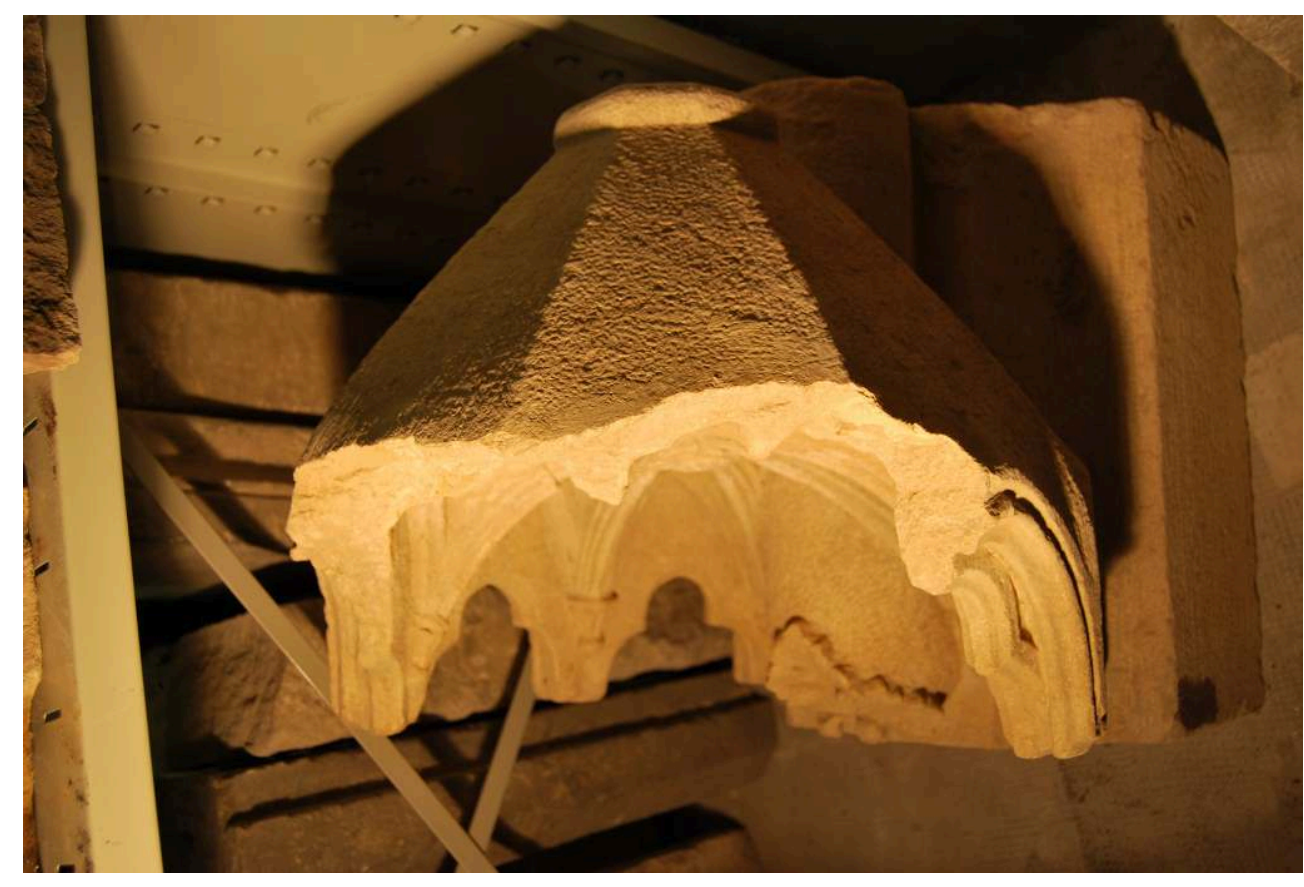

21 Certains morceaux sont imposants, comme le dais du trumeau central - long. $47 \mathrm{x}$ larg. 47 x prof. $72 \mathrm{~cm}$ (fig. 9) - ou les blocs provenant de l'oculus de la grande rose - le plus grand mesure $49 \times 19,5 \times 19,5 \mathrm{~cm}$ (fig. 6). Des pièces importantes sont conservées dans des collections particulières, notamment quatre grands morceaux de la demi-rose, dont les éléments trilobés mesurent plus d'un mètre de large ${ }^{18}$. Un autre élément du même ensemble est utilisé en remploi contre la façade d'une maison de Cluny ${ }^{19}$.

À l'occasion des travaux de restitution de la grande abbaye en 3D, entrepris en 2009 et 2010, la façade gothique a fait l'objet d'une recherche spécifique, ce qui a permis de vérifier nos hypothèses, sur la base des éléments numérisés. La courbure d'un bloc appartenant à l'oculus central (fig. 6) a permis de restituer la dimension exacte de cette partie et d'extrapoler le diamètre de la grande rose qui est de $8,958 \mathrm{~m}$ - Conant proposait $8,97 \mathrm{~m}^{20}$. La circonférence totale de la rose est de $28,18 \mathrm{~m}$, ce qui correspond à une largeur de 1,409 $\mathrm{m}$ au sommet de chacune des branches ${ }^{21}$. 
Fig. 6 - Cluny III, grande rose, fragment provenant de l'oculus central (cl. J. Rollier).

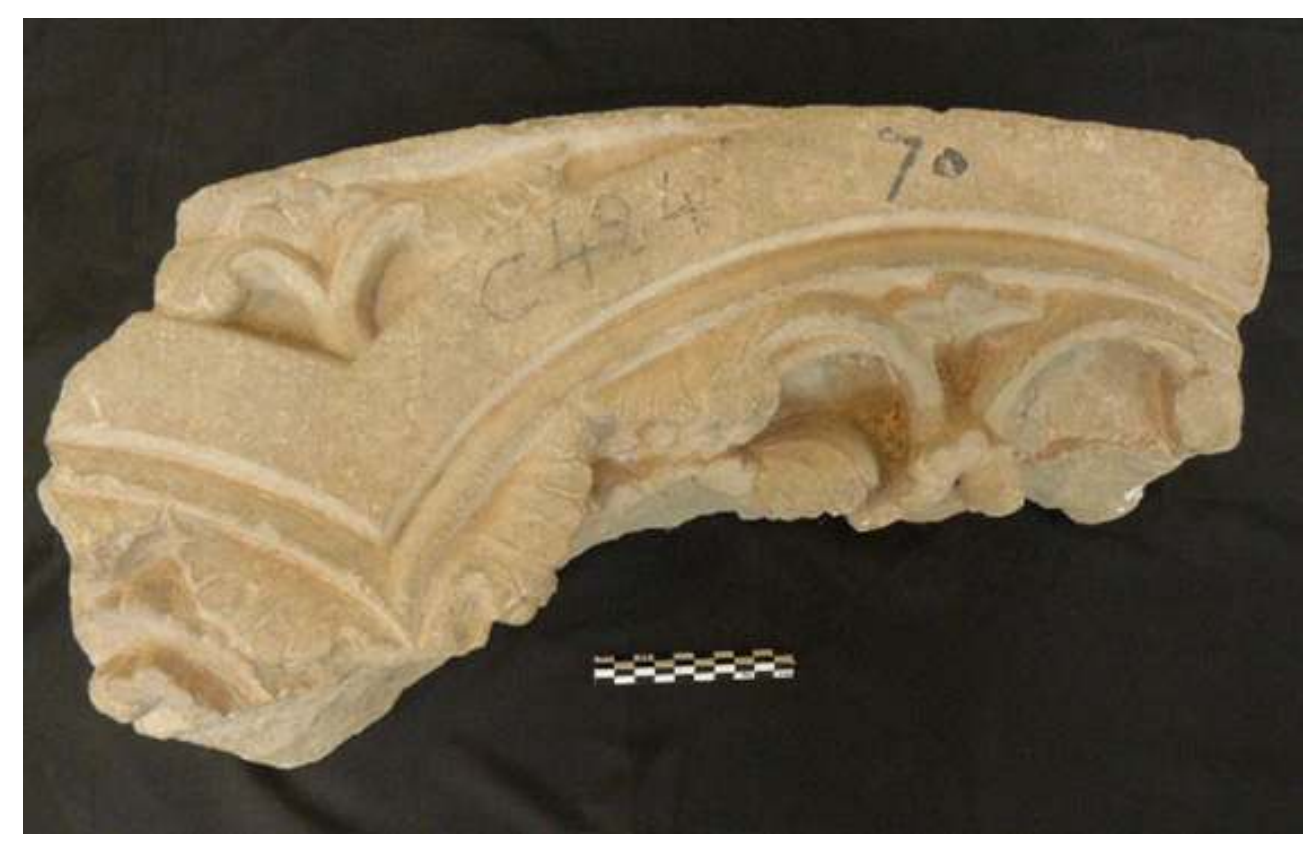

Selon le témoignage de Benoît Dumolin, la rose de Cluny comptait vingt branches ce qui est un nombre surprenant, la plupart des roses étant construite avec douze ou vingtquatre branches, en correspondance avec leur symbolique ${ }^{22}$. Certains oculus à huit compartimentsconstituent d'ailleurs peut-être des préalables à ces grandes roses ${ }^{23}$. Celle de la façade occidentale de Notre-Dame de Paris (fin XII ${ }^{\mathrm{e}}$ siècle) compte douze branches au centre et vingt-quatre branches en périphérie, pour un diamètre de près de dix mètres. La façade ouest de Chartres (vers 1216) s'ouvrait sur une rose à douze branches et douze médaillons, pour un diamètre de 13,36 $\mathrm{m}$. Le nombre présumé de vingt branches pour la rose de Cluny rappelle peut-être symboliquement l'organisation du Saint-Sépulcre, qui comporte vingt colonnes ${ }^{24}$.

La découverte de fragments de vitraux colorés et historiés à différents endroits de l'abbaye a conduit à une étude archéologique et historiographique de ces matériaux ${ }^{25}$. Pour le film destiné au public - produit par la société On-Situ ; fig. 11 -, le réalisateur a cherché à suggérer la présence de vitraux médiévaux dans toutes les parties de la grande abbatiale, en procédant à une sélection de verrières correspondant à la période envisagée. Pour la grande rose et la demi-rose de la façade gothique, notre choix s'est porté sur les vitraux de Chartres - transept sud, datés entre 1216-1227 - et Notre-Dame de Paris (vers 1268) ${ }^{26}$. Les branches sont ornées de motifs géométriques et végétaux répétitifs, tandis que les extrémités comportent des médaillons historiés. L'oculus de la grande rose est occupé par un Christ en majesté, sujet fréquemment représenté à cet emplacement. 
Fig. 11 - Cluny III, restitution virtuelle de la façade intérieure, image extraite du film Maior Ecclesia 2010 (Arts et Métiers ParisTech, On-Situ, CMN).

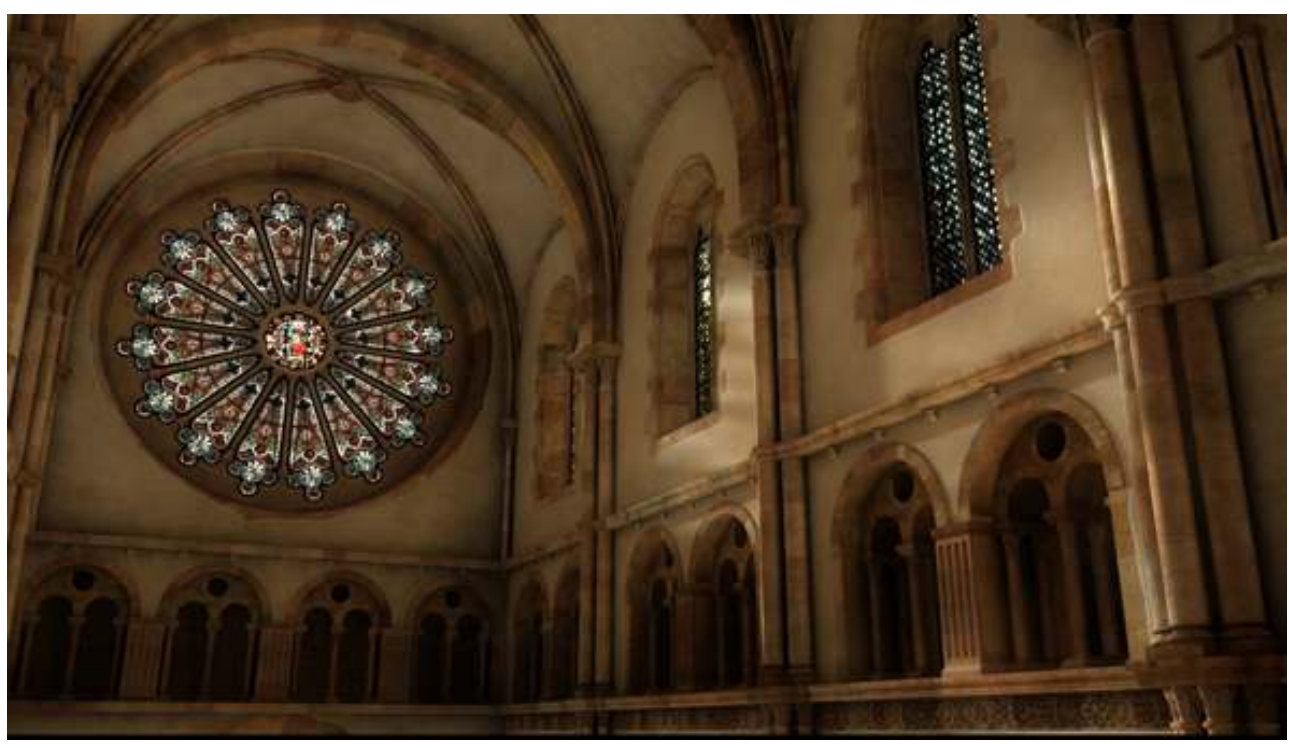

Au point de vue stylistique, l'étude de la façade gothique de Cluny III est un sujet extrêmement intéressant, qui demanderait une étude spécifique. Certains chapiteaux des piédroits peuvent être rapprochés des sculptures de Vézelay et de l'Auxerrois ${ }^{27}$. Les remplages de la demi-rose ressemblent aux décors de la cathédrale de Bourges (porche nord). Quant à la grande rose, à pétales trilobés, elle est peut-être dans la mouvance stylistique de la grande rose occidentale de Notre-Dame de Paris, ce qui situe la datation dans les années 1220-123028. L'étude des bases de colonnettes du piédroit apportera également des informations concernant l'atelier des sculpteurs de Cluny ${ }^{29}$.

\section{Étude des polychromies}

Parmi les cent soixante-dix fragments qui subsistent de la façade gothique, une trentaine conserve des restes polychromes. Plusieurs blocs provenant de la demi-rose présentent des restes pâlis d'un motif à chevrons rouge sur fond blanc, tel que nous en connaissons divers exemples en France $^{30}$, souvent repris aux $\mathrm{XIX}^{\mathrm{e}}-\mathrm{XX}^{\mathrm{e}}$ siècles, notamment à la cathédrale de Lausanne (1194-1220), dans le transept sud.

Un petit élément de l'oculus central de la grande rose présente un décor à chevrons ${ }^{31}$, très bien conservé, qui recouvre une polychromie plus ancienne (fig. 7). 
Fig. 7 - Cluny III, grande rose, fragment provenant de l'oculus central (cl. J. Rollier).

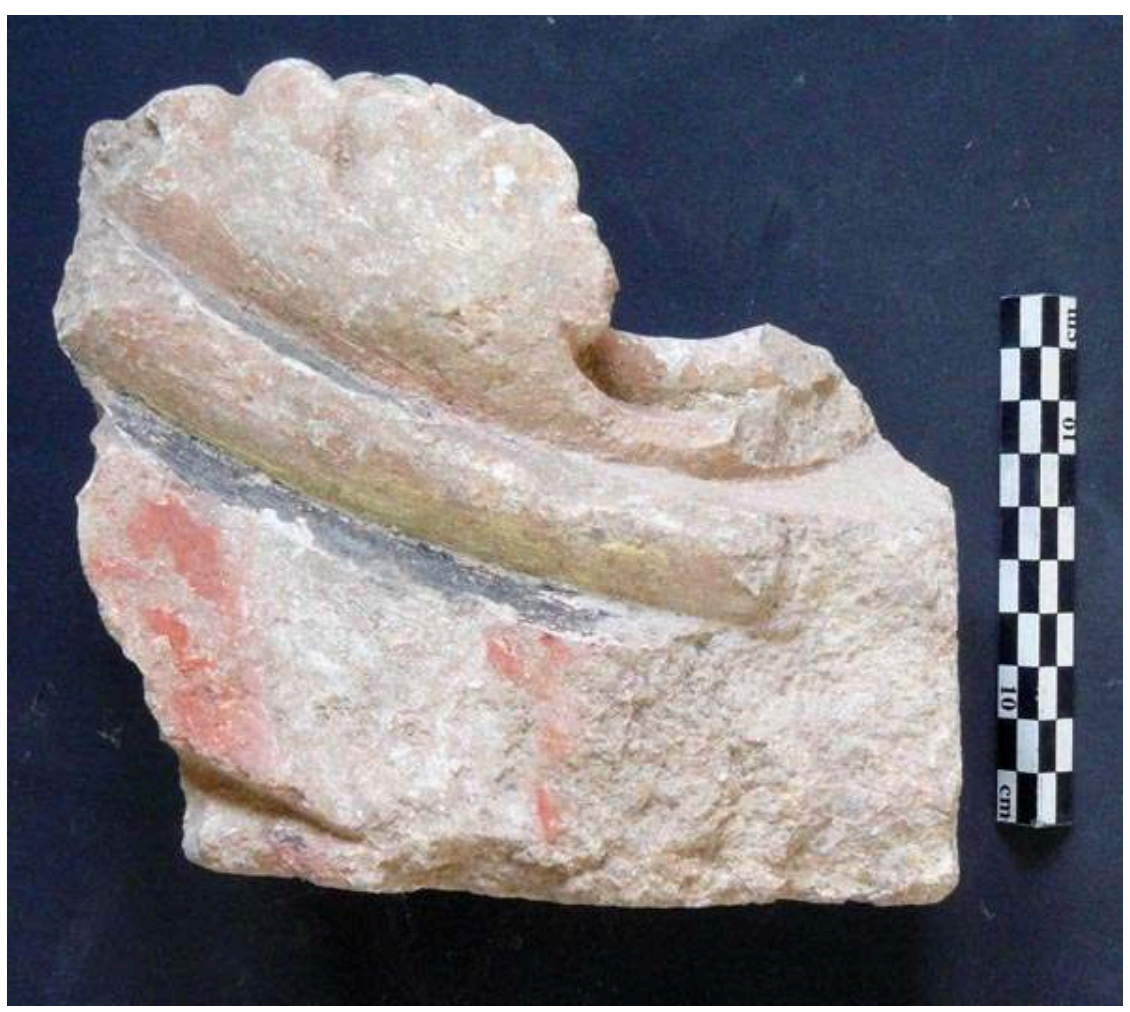

La stratigraphie indique une première coloration jaune, rouge et bleue, avec des fruits dorés à la feuille. Une seconde couche colorée comprend le décor à chevron rougeorangé sur fond blanc et semble avoir été réalisée selon une technique grasse (huile), qui se caractérise par un aspect légèrement satiné, des craquelures et des microboursouflures de matière. D'autres exemples de technique picturale "grasse » sont attestés, notamment à la cathédrale de Bourges, à Notre-Dame d'Étampes (vers 1140-1150) et Notre-Dame de Senlis (vers 1160-1170) ${ }^{32}$.

Les polychromies de Cluny, très pulvérulentes, ont été légèrement dépoussiérées et refixées ${ }^{33}$, excepté quelques éléments laissés vierges, de manière à permettre des analyses de liants. Divers prélèvements ont été analysés pour mieux connaître leur stratigraphie.

\section{Les tours Barabans : état de la recherche}

Les tours Barabans sont des constructions massives situées en avant de Cluny III, et qui encadrent la façade de son avant-nef. Lors des destructions révolutionnaires, les parties hautes des tours ont été abattues tandis que l'église était rasée presque dans son intégralité. L'abbaye mère clunisienne a suscité l'intérêt de nombreux chercheurs depuis le xix siècle en raison de son rayonnement européen et de ses très grandes proportions, mais le premier à avoir porté sur les vestiges un regard archéologique est l'américain K.J. Conant. Dès lors, comme en témoigne la Bibliotheca Cluniacensis Novissima publiée par l'université de Münster, la Maior Ecclesia d'Hugues de Semur est l'objet de réflexions multiples et continuellement renouvelées par les archéologues, historiens $\mathrm{s}^{34}$, historiens de l'art, mais aussi les ingénieurs travaillant aux reconstitutions $3 D^{35}$. 
31 Les Barabans, en revanche, n'ont pas été l'objet d'un tel engouement, bien que faisant partie intégrale de l'abbatiale : une datation tardive, une apparence austère et pauvre, à côté de la richesse des chapiteaux sculptés du chœur de Cuny III, les ont reléguées au second plan des analyses. C'est dans ce contexte que s'inscrit la recherche actuellement menée sur le sujet, par ailleurs motivée par les travaux de reconstitution 3D menés par l'équipe Gunzo. Une étude universitaire est en $\operatorname{cours}^{36}$, qui propose une hypothèse de restitution de leur élévation médiévale dans leur premier état, à partir des vestiges archéologiques et des sources textuelles, graphiques et iconographiques. Cette analyse comparative permet également de mettre en place une chronologie relative pour la construction des tours Barabans, par rapport à l'avant-nef, et de les replacer dans la chronologie absolue.

Le troisième point de réflexion est celui de la fonction des tours. Selon les sources descriptives, notamment les écrits de Benoît Dumolin ${ }^{37}$, en plus d'accueillir les cloches de l'abbaye, les tours servaient de salle aux archives (tour nord) et de lieu pour rendre la justice de l'abbé (tour sud). Ces rôles bien définis doivent faire l'objet d'une analyse avant d'être mis en relation avec la forme des tours. La question se pose, en effet, de la prédétermination de la fonction des tours en relation avec leur forme architecturale.

Les vestiges de chaque Baraban sont conservés sur une hauteur maximale d'environ $18 \mathrm{~m}$ à l'est, mais seulement sur 7,5 m à l'ouest, en raison de terrasses qui masquent les parements en partie basse. À partir de ces éléments, il est possible de réaliser une analyse archéologique du bâti des tours. Une distance de presque $10 \mathrm{~m}$ sépare les deux constructions de plan carré, d'environ $13 \mathrm{~m}$ de côté chacune. Leurs proportions, le grand appareil utilisé, le peu d'ouvertures et les contreforts à chaque angle en font des bâtisses massives. Elles ont changé de fonction au fil des siècles : aujourd'hui, la tour nord sert de dépôt archéologique et la tour sud est en cours de réhabilitation. De la face occidentale de cette dernière, il reste peu d'éléments d'origine, car son aménagement a nécessité le percement de nombreuses ouvertures. La tour nord est dans l'ensemble mieux conservée. L'intérieur est accessible et les parements médiévaux peuvent être observés. On pénètre à l'intérieur par le nord dans l'édifice divisé en deux niveaux, l'étage supérieur étant voûté.

Les sources écrites sur les tours Barabans sont peu nombreuses. Le Chronicon aliud cluniacense, texte rédigé par François de Rivo au $\mathrm{XV}^{\mathrm{e}}$ siècle, mentionne des réfections sur les tours ce qui fournit des dates ante quem pour leur construction, mais ne permet pas de datation précise. Plus tardivement, à la fin $\mathrm{du}$ XVIII ${ }^{\mathrm{e}}$ siècle, mais avant les destructions de 1789, Benoît Dumolin décrit l'abbatiale de Cluny d'ouest en est. Son travail est précieux, bien qu'il ne mentionne que l'extérieur des tours, parce qu'il est devant l'édifice qu'il décrit. À la même époque, Louis-Henri Lambert de Barive, avocat d'Autun, est envoyé dans la salle des archives de Cluny pour y copier des documents ${ }^{38}$, à la demande du cabinet des chartes, dont la création en 1764 a pour objet la collecte de documents témoignant des possessions monarchiques et nobiliaires en ces temps de troubles politiques ${ }^{39}$. De la même manière que Benoît Dumolin, il entreprend de décrire le lieu où il se trouve. La limite principale de ces deux documents est le vocabulaire descriptif employé au XVIII ${ }^{\mathrm{e}}$ siècle : instable d'un auteur à l'autre, mais également au sein des écrits d'un même auteur. Leurs descriptions architecturales sont à interpréter avec précaution.

Les tours ont fait l'objet de quelques représentations graphiques, la plupart du $\mathrm{XVIII}^{\mathrm{e}}$ siècle. Elles apparaissent soit sur des vues d'ensemble de l'abbatiale, soit dans des 
dessins de la façade de l'avant-nef. Elles n'ont jamais été représentées pour ellesmêmes. Les représentations d'Étienne Martellange - Veüe de l'Église de Cluni, 1617 - et de Jean-Baptiste Lallemand - "grande porte de l'église de l'abbaye de Cluny ", XVIII siècle - sont celles qui livrent les informations les plus fiables quant à la forme des tours avant leur destruction. Le premier, architecte jésuite, a parcouru la France dans le cadre de son travail et a réalisé pour son propre intérêt des dessins des monuments rencontrés : collèges, prieurés et abbaye. En tant qu'architecte, il a un regard acéré, comprend l'articulation des formes et est apte à les retranscrire, ce qui confère à son travail un grand intérêt architectural. En revanche, les dessins de Jean-Baptiste Lallemand sont réalisés dans le but d'illustrer un ouvrage de curiosités du patrimoine français. Ses représentations de monuments - il a également représenté des événements de la Révolution française, comme la prise de la Bastille - montrent toutes, au premier plan, de petites scènes de la vie quotidienne. Fabien Van Riesamburgh est le seul à avoir dessiné le revers de façade de l'avant-nef, en 1814, alors que l'abbaye était en cours de destruction. La mauvaise conservation du document le rend cependant difficile à exploiter.

Quelques plans de l'abbatiale permettent de travailler sur la disposition des tours par rapport aux autres bâtiments, ainsi que sur les accès. Le document le plus pertinent pour cette étude est également le plus ancien dont nous disposons. Il s'agit du plan dit Anonyme, daté d'environ 1700, qui représente l'ensemble de l'abbaye avant sa destruction et permet de mettre en lien les bâtiments entre eux.

Les vestiges ont fait l'objet d'une série d'observations archéologiques des parements, de leurs remaniements et des liens architecturaux entre les murs. Chaque tour présente sur son parement extérieur et en partie basse un grand appareil de pierres de taille, présentant une alternance d'assises allongées et régulières, surmonté d'un appareil dont les modules taillés sont plus petits. Ils correspondent à un premier état de construction des tours. À l'intérieur, le parement est constitué d'un petit appareil de moellons éclatés. La césure horizontale entre les deux appareils de pierres taillées se retrouve sur les contreforts. Elle peut témoigner d'un arrêt de chantier, plus ou moins long, pour raisons financières ou manque d'approvisionnement par exemple, ou simplement d'un changement de parti dans la construction. Centrées sur les faces ouest et est de la tour nord, deux fines baies en plein cintre sont conservées juste sous la toiture actuelle et, ne présentant pas de césure d'insertion, appartiennent au parement médiéval de grand appareil. Sur la face sud, seuls l'appui et les premières assises des piédroits d'une troisième baie sont conservés.

Ces éléments architecturaux observés sur les vestiges sont un moyen de juger de la fiabilité des sources, selon la manière dont ils sont décrits ou représentés. Benoît Dumolin, par exemple, a parfaitement repositionné les tours par rapport à la façade, décrit leur forme carrée et les contreforts. Nous pouvons alors considérer que sa description est proche de la réalité et nous appuyer dessus pour restituer les parties disparues des tours. L'auteur mentionne deux «cordons" qui entouraient chaque Baraban : le premier, couronnant les contreforts, qui n'atteignaient pas le sommet, et le second faisant la «base des vitraux ». Sa description n'est que peu détaillée, mais nous apprend que chaque face était rythmée horizontalement par un bandeau et qu'il y avait au minimum un second niveau percé de baies.

Les illustrations des XVII ${ }^{e}$ et XVIII ${ }^{e}$ siècles dont nous disposons sont analysées de la même manière, afin d'être exploitées au plus juste. Prenons l'exemple de la représentation de 
la façade de l'avant-nef par J.-B. Lallemand ${ }^{40}:$ y figurent, pour la façade, les éléments observés sur les vestiges, ainsi que ceux décrits par Benoît Dumolin pour les parties hautes. Cependant, la baie, dont nous avons observé les parties basses sur la face sud de la tour nord et qui appartient au premier état médiéval, ne figure pas sur le dessin. En effet, les faces intérieures des tours sont nues et dépourvues de baies selon J.B. Lallemand, ce qui surprend d'autant plus, que, pour diffuser le son des cloches, le dernier niveau était sans aucun doute très ouvert. La gravure de P.-F. Giffart ${ }^{41}$, datée d'environ 1713 et représentant l'abbatiale vue du nord, montre les faces septentrionales et orientales percées de baies, de même que la représentation d'É. Martellange,qui offre le même point de vue. De fait, J.-B. Lallemand a été conduit à faire des choix pour mettre en valeur certains éléments au détriment d'autres, absents de ses représentations. Ainsi, il a représenté de manière très détaillée la rose, le portail et les faces occidentales des tours, mais a en revanche mis de côté les éléments du second plan, qu'il a jugé moins importants.

À partir de la documentation graphique, iconographique et des vestiges archéologiques, il est possible de proposer une restitution du premier état de construction des Barabans : les deux tours de plan carré, massives, sont encadrées à chaque angle d'un contrefort, qui s'interrompt avant le sommet et est couronné d'un bandeau. Leur hauteur, identique, est comprise entre 40 et $45 \mathrm{~m}^{42}$. Un second bandeau le surmonte. Le premier niveau de la tour nord est percé sur chaque face d'une baie plein cintre, étroite et centrée, que nous pouvons encore observer aujourd'hui. Audessus de celle-ci, les faces orientale et occidentale possèdent une seconde baie identique. Seule la face occidentale de la tour sud est percée par cette même baie. Entre les deux bandeaux, cette tour ne possède aucune ouverture, tandis que sa voisine est percée d'une nouvelle baie, identique aux précédentes, sur chaque façade. Le dernier niveau d'élévation est percé pour chaque tour de deux baies plein cintre, disposées l'une à côté de l'autre. La tour nord est crénelée, mais la tour sud présente une structure différente de croisillons de bois, illustrés par J.-B. Lallemand. Selon les plans, la tour de la justice est ouverte au sud et à l'ouest. Ces données ne sont pas vérifiables en raison du réaménagement en habitation du bâtiment. L'accès à la tour nord fait l'objet d'une réflexion plus poussée, car il pointe les questions de circulation de l'abbé depuis le palais abbatial jusqu'au sanctuaire. En effet, la face septentrionale, et plus précisément la salle des archives, est reliée au logis de l'abbé par un corridor, comme en témoigne L.-H. Lambert de Barive. Cet accès au nord existait-il avant la construction du palais abbatial de Jean de Bourbon? Il existe, en outre, très certainement, un passage ouvrant sur l'avant-nef depuis la tour, au moyen d'un escalier.

41 Nous pouvons donc restituer quatre niveaux d'élévation à partir des élévations extérieures, mais il est difficile de dire s'ils correspondent à leur organisation intérieure. La tour nord possède au minimum deux étages, un pour la salle des archives et un autre pour les cloches. En revanche, aucun élément ne nous renseigne pour la tour sud.

42 Les observations archéologiques ont également permis de proposer une chronologie relative de construction du massif occidental de Cluny III. Les liaisons entre l'avant-nef et son portail et les tours Barabans témoignent d'une construction continue. Ainsi, les parties basses des tours ont été élevées parallèlement à l'achèvement de la dernière travée occidentale de l'avant-nef, c'est-à-dire entre la fin $\mathrm{du} \mathrm{XII}^{\mathrm{e}}$ et le début du XIII ${ }^{\mathrm{e}}$ siècle, selon Gilles Rollier et Nadine Roiné qui ont fouillé cette dernière entre 1988 
et $1990^{43}$. Une mention dans le Chronicon aliud cluniacense révèle que l'une des tours fut portée à hauteur de la seconde sous l'abbatiat de Pierre II de Chastellux (1322-1344) ${ }^{44}$. Ainsi, la construction des Barabans ne s'est pas faite en une seule fois, malgré leur élévation très semblable : seuls diffèrent le nombre d'ouvertures et le couronnement.

Les travaux de Sébastien Barret portant sur les archives clunisiennes nous éclairent sur la fonction de la tour nord ${ }^{45}$. Celle-ci est en effet nommée turris privilegiorum dans un texte de 1403. Il s'agit de la première occurrence du lieu de conservation des archives de l'abbaye, qui représentent son bien le plus précieux. Dès le début du XIv ${ }^{\mathrm{e}}$ siècle, des écrits mentionnent un lieu réservé aux documents garantissant les privilèges clunisiens, mais sans préciser leur lieu de stockage. S. Barret propose plusieurs hypothèses concernant la date d'installation des archives dans la tour nord, la plus probante, selon lui, étant l'établissement d'un lieu fixe d'entrepôt lors de la restructuration au milieu XIII ${ }^{\mathrm{e}}$ siècle. Il est impossible de savoir avec certitude si la tour fut édifiée de cette manière dans le but précis d'abriter les archives. Son apparence austère et peu ouverte sur l'extérieur ne permet pas d'y exécuter les rituels monastiques, messes ou autres cérémonies, en revanche, elle est tout à fait adaptée à la protection d'objets ou documents précieux à l'abbaye. La forme de la tour n'est pas due au culte et il est possible qu'elle la doive à sa fonction, mais nous verrons qu'une autre interprétation est possible.

Des descriptions tardives des $\mathrm{XVII}^{\mathrm{e}}$ et $\mathrm{XVIII}{ }^{\mathrm{e}}$ siècles révèlent l'utilisation de la tour sud comme lieu de justice et d'enfermement ${ }^{46}$. Sa présence à l'entrée du monastère n'est pas une exception: à Tournus, monastère clunisien voisin de son abbaye mère, des bâtiments réservés à l'exercice de la justice sont édifiés près de l'entrée au XIII ${ }^{\mathrm{e}}$ siècle $^{47}$. De la même manière, à Saint-Denis, la justice était exercée dans des locaux situés près de l'enceinte ${ }^{48}$. De même, nous ne pouvons affirmer que la forme de la tour a été conditionnée par l'usage qui en était fait, mais, à nouveau, elle est totalement adaptée à cette fonction.

45 À l'aide des Écritures, des hypothèses peuvent être faites quant à la source de cette architecture. Les théologiens médiévaux ont transposé sur le lieu de culte chrétien, l'Église, l'image de la Jérusalem céleste. L'Apocalypse constitue une source d'interprétation : la sainte cité, lors de sa descente sur terre, était « entourée d'une très haute muraille » (Ap 21). À la manière des édifices à façade harmonique, comme SaintÉtienne de Caen, les tours de façade de Cluny III renvoient à cette image de fortification, tant par leur forme que par leur disposition en avant de la façade, qui se veut comme un rappel de l'entrée dans la cité de Dieu. Cette architecture est un renvoi direct aux formes carolingiennes, étudiées au $\mathrm{xx}^{\mathrm{e}}$ siècle par Carol Heitz: les églises à cette époque étaient précédées à l'occident d'un atrium, dont l'accès par trois côtés menait à la façade surmontée de deux, parfois trois tours, celle au centre étant la plus haute ${ }^{49}$. Dans une chapelle haute étaient célébrés les offices de Pâques et de Noël : elle était de ce fait réservée au culte du Christ. Ainsi, les tours Barabans s'inscrivent par leur forme à la suite d'une longue tradition architecturale, qui prend sa source dans le texte de l'Apocalypse, à travers l'image de la Jérusalem céleste.

Ce travail de recherche sur les tours Barabans a permis de révéler leur forme originelle, tout du moins de s'en approcher au plus près. Des chronologies relatives et absolues ont été mises en place à partir d'observations archéologiques, qui seront affinées par l'étude du bâti - relevés archéologiques, analyse des matériaux... Plus importantes encore sont les nouvelles questions que suscite cette première approche, notamment 
autour de la circulation à l'entrée de la grande abbatiale : n'y avait-il qu'un seul accès à la salle des archives depuis le palais abbatial? Quel en était l'accès avant la construction du palais? Comment la circulation se faisait ou ne faisait-elle pas entre tours et avant-nef? Pouvait-on passer d'une tour à l'autre en circulant «dans » la façade? En outre ces questions invitent à repenser leur organisation intérieure.

\section{NOTES}

1. D. RICHE, L'ordre de Cluny à la fin du Moyen Âge, Saint-Étienne, 2000.

2. Une étude existe sur la chapelle Jean de Bourbon : B. MAURICE, « Le décor sculpté de la chapelle funéraire de Jean de Bourbon, abbé de Cluny ", Actes des journées internationales Claus Sluter, Dijon, 1992, p. 151-164.

3. J. Rollier-Hanselmann, responsable restitution architecturale, équipe Gunzo, Arts et Métiers Paristech Cluny [http://www.cluny-numerique.eu]; C. CollomB, Les tours Barabans en façade de l'abbatiale de Cluny III, mémoire de master 1, université de Lyon 2, 2010 et G. FERDEK, Étude du portail gothique de l'église de Cluny III : polychromie, restauration, restitution, mémoire de master 1, université de Bourgogne, Dijon, 2010.

4. G. ROLLIER et N. ROINÉ, Narthex de Cluny III et cour abbatiale, rapport de synthèse, fouilles de sauvetage programmé, 1988-1990 ; K. J. CONANT, Cluny. Les églises et la maison du chef d'ordre, Mâcon, 1968 ; A. BAUD, Cluny, un grand chantier médiéval au cœur de l'Europe, Paris, 2003.

5. K. J. CONANT, Cluny..., ibid.

6. K. J. Conant, Cluny..., ibid., p. 129. Biblioteca cluniacensis, col. 1679.

7. B. MARGUERY-MELIN, «La destruction de l'abbaye de Cluny, 1789-1823 », Bulletin du Centre d'études clunisiennes, 1985, p. 39-41.

8. K. J. CONANT, Cluny..., op. cit., pl. XIII-XIV.

9. J.-B. LALlemAND, « Grande porte de l'église de l'abbaye de Cluny », vers 1773, dessin conservé au musée d'art et d'archéologie de Cluny, et gravé par Niquet pour illustrer le Voyage pittoresque de la France (t. 2, 1748, pl. $\mathrm{n}^{\circ} 56$ ).

10. K. J. Conant, Cluny..., op. cit., p. XXIV.

11. A. DE THOISY, Sagot, mémoire de Bourgogne, dessins et lithographies du XIX siècle, musée d'art et d'archéologie de Cluny, Cluny, 1994.

12. Le manuscrit a été donné par M. Ph. de Champeaux, qui le tenait lui-même d'un ami clunisois. Nous remercions le généreux donateur pour ce document ; N. STRATFORD, «Fondation de la Sacrée Abbaye de Cluny ", Mémoires de la Société Éduenne des lettres, sciences et arts, 56/3 (1999-2000), p. 387-388.

13. B. Dumolin, Histoire et description de la ville et des environs de Cluny, archives communales de Cluny, musée d'art et d'archéologie, ms. 71, 1749-1778.

14. G. ROLLIER, Fouilles de sauvetage programmé, 1988-1990, Narthex de Cluny III et cour abbatiale, Cluny, 1991. Le portail roman est sculpté dans les calcaires oolithiques et le calcaire blanc de la Lie.

15. G. ROLLIER, "Les fouilles archéologiques de l'avant-nef », Cahiers du musée d'art et d'archéologie de Cluny, juillet 1996, p. 16-21.

16. Fragment $n^{\circ} 75.1 .2$ (haut. $32 \times$ larg. $48,5 \times 59,5 \mathrm{~cm}$ ) découvert lors de la démolition des bâtiments accolés à la tour nord des Barabans (fiche musée). 
17. $\mathrm{N}^{\circ}$ inv. 49.3.1 provenant d'un propriétaire privé (fonds ancien du musée).

18. J.-D. SALVÊQUE, "Quelques témoins de l'architecture religieuse à Cluny », Bulletin du Centre d'études clunisiennes, 1992, p. 42-43 ; K. J. Conant (Cluny..., op. cit., pl. XCIX, fig. 230) a publié une image d'un de ces éléments conservés à Nancelles, mais il existe trois autres fragments malheureusement envahis par les lichens et les mousses. Les pièces mesurent respectivement 105,110 et $135 \mathrm{~cm}$ de large pour une hauteur de $60 \mathrm{~cm}$ environ.

19. Documentation J.-D. Salvêque.

20. Voir nos articles précédents dans le Bucema, 13 (2009), p. 157-170 [http://cem.revues.org/ index11058.html] et 14 (2010), p. 235-250 [http://cem.revues.org/index11601.html]. À l'occasion du $1100^{e}$ anniversaire de l'abbaye de Cluny, des financements exceptionnels ont permis d'étudier et de numériser certains fragments lapidaires, en vue de la restitution virtuelle en 3D.

21. Restitution 3D par Guillaume Schotté, ingénieur Arts et Métiers ParisTech, équipe Gunzo.

22. P. coWEn, Roses médiévales, Londres, 1979 ; N. HISCocK, The Symbol at your door, Number and geometry in Religious architecture of the Greek and Latin Middle Ages, Aldershot, 2007, p. 273-282.

23. Oculus à huit compartiments de Barfreston (Kent). P. cowEN, Roses médiévales..., ibid.

24. N. HIScock, The Symbol..., op. cit., p. 237.

25. Ce matériel est en cours d'étude et d'analyse.

26. P. COWEN, Roses médiévales..., op. cit.

27. A. TIMBERT, Vézelay, Le chevet de la Madeleine et le premier gothique bourguignon, Rennes, 2009.

28. Voir F. JOUBERT, La sculpture gothique en France, XII ${ }^{e}$-XIII ${ }^{e}$ siècle, Paris, 2008, fig. 38-39 ; A. TIMBERT, Vézelay..., op. cit.

29. Voir, notamment, M. TRICoIT, «Remarques sur l'évolution de la base de colonne au XIII ${ }^{\mathrm{e}}$ siècle en France du Nord: maturité et disparition du profil attique », Bulletin de la Société des fouilles archéologiques et des Monuments historiques de l'Yonne, 23 (2006), p. 17-25.

30. E. VIOLLET-LE-DUC, Dictionnaire raisonné de l'architecture française du XI ${ }^{e}$-XVI $I^{e}$ siècle, 1854-1868.

31. Fragment IX.40 découvert lors des fouilles de G. Rollier, entre 1988-1989.

32. R. ROSSI MANARESI, «Observations à propos de la polychromie de la sculpture monumentale romane et gothique ", in D. VERRET et D. STEYAERT (dir.), La couleur et la pierre. Polychromie des portails gothiques, Paris, 2002.

33. Un dépoussiérage au pinceau doux a été suivi d'un refixage des couleurs. Plusieurs essais de fixatifs ont été testés préalablement. Nous avons utilisé du Paraloid B72 dilué à $5 \%$ dans un mélange d'acétone/white-spirit (25 g acétone ; $75 \mathrm{~g}$ white-spirit).

34. Par exemple: A. BAUD, Cluny, un grand chantier médiéval au cœur de l'Europe, Paris, 2003 ; D. IOGNA PRAT, Études clunisiennes, Paris, 2002 ; N. REVEYRON (dir.), Hugues de Semur 1024-1109. Lumières clunisiennes. $1100^{e}$ anniversaire de l'abbaye de Cluny, IX centenaire de la mort d'Hugues de Semur, 6e abbé de Cluny, Paray-le-Monial, 2009; C. SAPIN, "Cluny II et l'interprétation archéologique de son plan ", in D. IOGNA-PRAT et J.-C. PICARD (éd.), Religion et culture autour de l'an Mil, Paris, 1990, p. 85.

35. Équipe Gunzo dirigée par Christian Père.

36. C. CollomB, Les tours Barabans..., op. cit., p. 24-28 et 33-39.

37. B. DUMOLIN, Description..., op. cit., f. 18.

38. S. BARRET, «La Tour des archives de Cluny », Livraison d'histoire de l'architecture, 10 (2005), p. 9-17.

39. O. PARIS-BARUBÉ, « Remuements de chartes et passage à l'histoire : la fatalité du déficit de récit dans les programmes centralisés de collecte de sources (1750-1850) », inP. POIRRIER et J. LAUVERNIER (dir.),Historiographie et archivistique. Écriture etméthodes de l'histoire à l'aune de la mise en archives [http://tristan.u-bourgogne.fr/UMR5605/publications/historiographie/O_Paris-Barube.html].

40. J.-B. LALLEMAND, « Grande porte... », op. cit. 
41. P.-F. GIFFART, Conspectus ecclesiae cluniacensis, plan et vue depuis le nord de l'église de Cluny III, musée d'art et d'archéologie de Cluny.

42. 140 pieds d'après B. Dumolin : selon l'unité qu'il utilise, pied romain ou pied du roi, la hauteur exacte varie. De plus, cette mesure est probablement déjà une approximation. Il est face aux tours lorsqu'il les décrit: les 140 pieds désignent-ils la hauteur depuis la terrasse ou depuis le niveau de sol de l'avant-nef?

43. G. ROLLIER et N. ROINE, Narthex de Cluny III..., op. cit.

44. F. DE RIVo, Chronicon cluniacensis, in Dom MARRIER et A. DUSCHESNE (éd.), Bibliotheca Cluniacensis, 1614 (réédition Protat, Mâcon, 1915), col. 167.

45. S. BARRET, La mémoire et l'écrit : l'abbaye de Cluny et ses archives ( $\mathrm{x}^{\mathrm{e}}$-XVIII ${ }^{\mathrm{e}}$ siècle), Münster, 2004 (Vita regularis, 19).

46. Dom Mabillon cité par K. J. Conant inCluny..., op. cit., p. 24 ; B. Dumolin, Description..., op. cit.

47. B. SAINT-JEAN VITUS, Tournus: le castrum, l'abbaye, la ville, XI ${ }^{e}-\mathrm{XIV}{ }^{e}$ siècles et prémices: analyse archéologique d'un développement monastique et urbain, thèse de doctorat, université de Lyon 2 , 2006, 7 vol.

48. J. FORMIGÉ (dir.), L'abbaye royale de Saint-Denis, recherches nouvelles, Paris, 1960.

49. C. HEITZ, Recherche sur les rapports entre architecture et liturgie à l'époque carolingienne, thèse pour le doctorat de $3^{\mathrm{e}}$ cycle, École pratique des hautes études, Paris, 1963.

INDEX

Index géographique : France/Cluny

Mots-clés : abbaye, façade gothique

\section{AUTEURS}

\section{GAËLLE FERDEK}

Collaboratrice 